\title{
Calculation of pipe residual lifetime of the exploited oil and gas pipelines
}

\author{
Ivan Shtoyko, Iryna Dolinska, Yaroslav Khaburskyi, Yurii Kaniuk \\ Karpenko Physico-Mechanical Institute of the NAS of Ukraine, Ukraine, Lviv, 5 Naukova St, \\ E-mail: ivan.shtoyko@gmail.com
}

The calculations of the residual lifetime of long-term exploited pipes of oil and gas pipelines for X52, X60 and $X 70$ steels are presented taking into account their hydrogenation from internal pipe side and ground corrosion from the external side. It is shown that steel degradation decreases essentially pipe residual lifetime.

Keywords - gas and oil pipelines, calculating methods for determining pipe residual lifetime, corrosion and hydrogen degradation of pipe, the residual lifetime of pipes, energy approach, hydrogen concentration, stress hydrogen cracking, stress corrosion cracking.

\section{Introduction}

Long-term operation of oil and gas transit pipelines causes degradation of as-received mechanical properties what intensifies hydrogen-induced and stress corrosion cracking and finally failure risk increase. From the other hand, it is known [1-4] that the parameters of crack growth resistance (the parameters of fracture mechanics) are the most sensitive to in-service degradation, especially the corrosion crack growth characteristics. Usually, the factor of material degradation does not take into consideration at a modelling of crack growth under the simultaneous action of working stresses and aggressive environments. In this paper, the methods of an evaluation of the residual lifetime of pipes of oil and gas pipelines with taking into account their degradation during operation are presented using corrosion and hydrogen fracture mechanics approaches. The results of such calculations are given for some cases of crack growth across the pipe wall.

\section{Evaluation of X52 steel pipe of the gas pipeline residual lifetime under static pressure and ground corrosion action}

Consider a pipe with pipe wall thickness $h=12 \mathrm{~mm}$ of X52 steel, which was subjected to a long-term static pressure of natural gas $p=8 \mathrm{MPa}$, and which weakened by external surface semielliptical crack with semi axes $a_{0}, b_{0}$ (Fig. 1). It is considering, that soil environment fills a crack and caused its corrosion-mechanical propagation.

We assume that pipe material is degraded during operation before and during corrosionmechanical crack propagation. The task consists in the determination of time $t=t_{*}$ which needed to crack propagation through the pipe wall i.e. $b=h$ and its decompression.

According to experimental research [5], a crack in such steel propagates under long-term static loading and soil corrosion mainly with a constant rate $V_{k}$. In this case, for non-operated pipe the rate is $V_{k}(0)=1,03 \cdot 10^{-3} \mathrm{~m} /$ year and for steel after 30 years operation it is $V_{k}(30)=8,03$. $10^{-3} \mathrm{~m} /$ year.

Based on this experimental data for random operation time of pipe made of X52 steel the equation for approximate determination of the rate $V_{k} \approx V_{k}(t)$ could be written as:

$$
V_{k}(t)=10^{-3}\left[1,03+0,23\left(t_{0}+t\right)\right](\mathrm{m} / \text { year })
$$


where $t_{0}$ - pipe operation time before gas pipeline residual lifetime estimation.

The problem is solved by using the energetic approach [6-8] and in this case, its reduced to mathematical model:

$$
\begin{gathered}
\frac{\partial \rho}{\partial t}=V_{k}(t) \sqrt{1+\rho^{-2} \frac{\partial^{2} \rho}{\partial \alpha^{2}}} \\
t=0, \rho(0, \alpha)=\rho_{0}(\alpha) ; t=t_{*}, \rho\left(t_{*}, \pi / 2\right)=h .(2)
\end{gathered}
$$

$\alpha, \rho$ - coordinates of polar system $O \alpha \rho$ which determine kinetic contours system of corrosionmechanical crack. The equation (2) with initial and final conditions is nonlinear partial differential equations and its solution associated with some mathematical difficulties. Therefore, to get approximate the solution of this problem we proceed as follows. Science initial crack is semi-elliptical and its propagation rate is constant than it could be assume that crack would slightly differ in its propagation from the elliptical form. In this regard we consider that crack in its propagation will be semi-elliptical and thus the solution of equation (2) reduces to differential equations system:

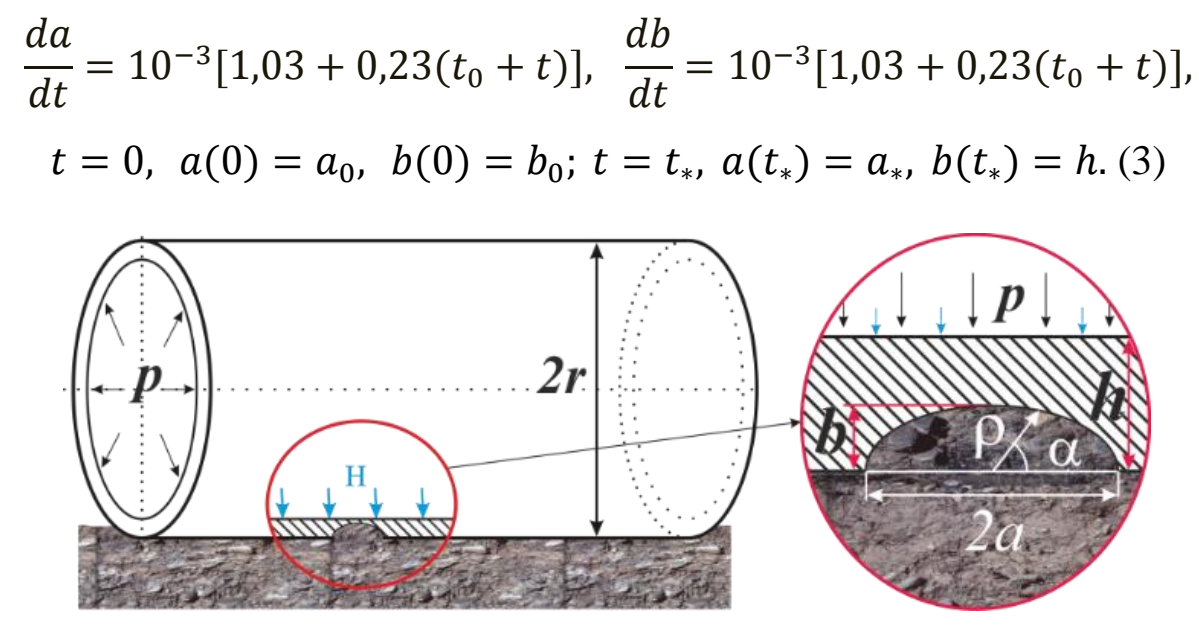

Fig.1. Loading scheme of pipe with an external crack under soil environment action

Solving the system of differential equations (3) with initial and final conditions for determination kinetic changes of semi-axes contours of the crack system we will get correlation:

$$
\begin{aligned}
& a(t)=a_{0}+10^{-3}\left(1,03 t+0,23 t t_{0}+0,01 t^{2}\right), \\
& b(t)=b_{0}+10^{-3}\left(1,03 t+0,23 t t_{0}+0,01 t^{2}\right) .
\end{aligned}
$$

To determine pipe residual lifetime following equation is used:

$$
t_{*}=-0,5\left(103+23 t_{0}\right)+\sqrt{0,25\left(103+23 t_{0}\right)^{2}+10^{5}\left(h-b_{0}\right)}(5)
$$

According to Eq. (5) dependences of pipe residual lifetime $t_{*}$ on initial crack depth and pipe operation time $t_{0}$ are built (Fig. 2)

Value $t_{*}$ in this case determined by equation (5) and it is equal to $t_{*}=9,76$ year. The kinetic contour system of semi-elliptical crack propagation with initial crack size $a_{0}=0,002 \mathrm{~m}, b_{0}=$ $0,001 \mathrm{~m}$ and $t_{0}=0$ is shown on figure 3 . 
Based on obtained results it could be concluded that pipe residual lifetime strongly depends on its initial operation time and crack contour approaches to circular one.

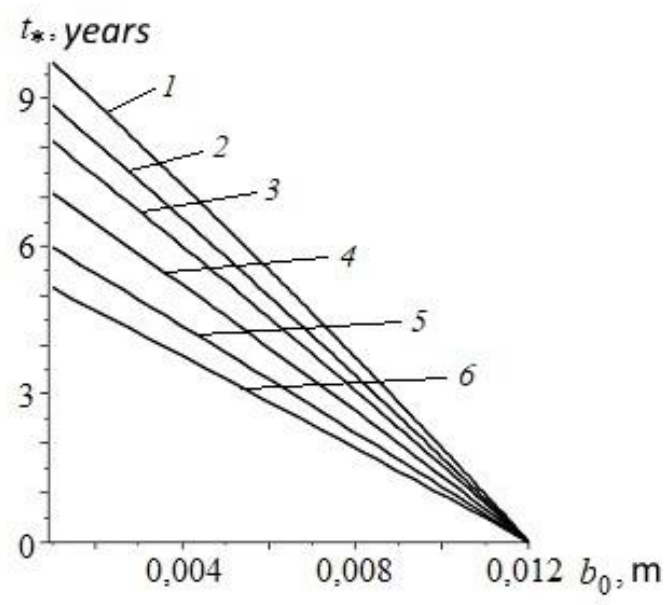

Fig.2. Dependence of pipe residual lifetime $t_{*}$ on initial crack depth b0 and pipe operation time $t_{0}$ : $1-t_{0}=0 ; 2-4 ; 3-8 ; 4-15 ; 5-25 ; 6-35$.

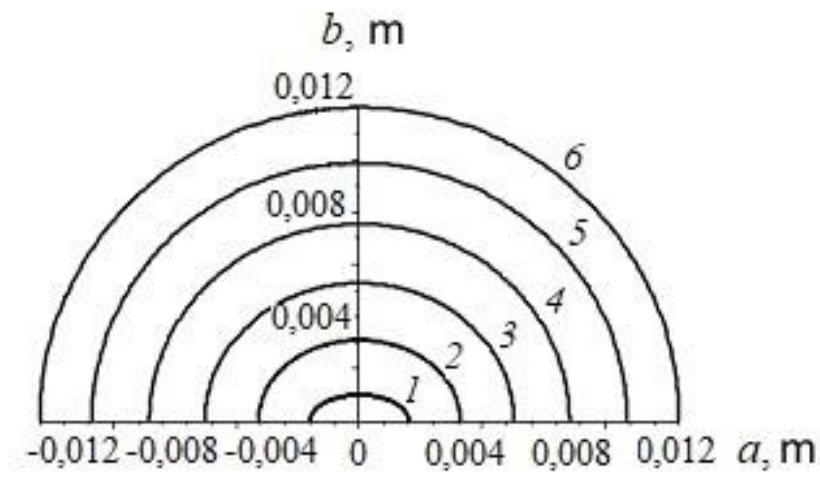

Fig.3. Crack contour kinetic for different time: $1-t=0 ; 2-2 ; 3-4 ; 4-6 ; 5-9 ; 6-9,76$

\section{Evaluation of residual lifetime for X70 steel pipe of the oil pipeline at oil turbulence}

In many cases, oil pipeline fracture begins with pipe internal surface where surface cracks are initiated as a result of steel degradation. It is well known that under turbulent oil flow pipe is subjected to dual-frequency loading: high frequency $\omega_{1}=0,6$ second $^{-1}$ (period of cycle $T_{1} \approx 1,7$ second) is caused by turbulent oil flow; low frequency $\omega_{2}=2,1 \cdot 10^{-6}$ (period of cycle $T_{2} \approx$ 476190 the second) - as a result of pumping oil stops, by shutting off the pumps, closing the latches, etc. That is in one cycle of low frequency $N_{1}$ fluctuation of high frequency is passed. Such a dualfrequency type of loading is taking into account in residual life-time of the oil pipeline calculation. The core of residual life-time of oil pipeline calculation in this case is as follows.

Considering oil pipe, radius $r_{3}=710 \mathrm{~mm}$ and wall thickness $h_{1}=18,7 \mathrm{~mm}$ made of steel X70 with internal surface semi-elliptical crack, in which turbulent oil flow is moving under pressure $p \approx$ $4 \mathrm{MPa}$ and turbulence emission $\Delta p \approx 0,25 \mathrm{MPa}$. At the same time, the pressure change in a pipe changes according to dual-frequency law. The task consists in the determination of the oil pipeline 
residual life-time namely the number of loading cycles of low-frequency $N=N_{*}$ after which the crack contour passed through pipe wall and its decompression starts.

Based on the energy approach such a problem solution reduces to the nonlinear partial differential equation. To approximately determine pipe residual lifetime with sufficient accuracy for engineering purposes well known equivalent areas method is used. In this case, the task solution reduces to following mathematical model:

$$
\begin{aligned}
& \frac{d \rho}{d N}=\frac{\alpha(1-R)^{4}\left[K_{\operatorname{Imax}}^{4}\left(1+N_{1}\left(1-R_{1}\right)^{4}\right)-K_{t h}^{4}\right]}{\left(K_{f C}^{2}-K_{\operatorname{Im} a x}^{2}\right)} \\
& N=0, \rho(0)=\rho_{0}=\sqrt{a_{0} b_{0}} ; N=N_{*}, \rho\left(N_{*}\right)=h_{1}(6)
\end{aligned}
$$

$\rho$ - is a radius of semi-elliptical crack the area of which is equal to the area of real crack; $\alpha, K_{t h}, K_{f C}$ - is a characteristics of the fatigue crack grow diagram; $R, R_{1}$ - coefficients of cycle asymmetry under low- and high-frequency loading correspondently $\left(R=0,1 ; R_{1}=0,94\right) ; K_{\text {Imax }}$ - SIF maximum value in one cycle and on a semi-elliptical crack contour.

However, during operation, the pipe material is degraded i.e. parameters $\alpha, K_{t h}, K_{f C}$ will be depended on operation time $t$ and changed in the direction of reducing its fatigue fracture resistance. The characteristics dependency $\alpha, K_{t h}, K_{f C}$ on time we consider as linear and on this base, the correlation could be written as:

$$
\begin{gathered}
\alpha(N)=10^{-8}\left[1,6-2 \cdot 10^{-4}\left(N+N_{0}\right)\right](M P a)^{-2}\left(\text { cycle }^{-1}\right. \\
K_{t h}(N)=9,1-1,3 \cdot 10^{-3}\left(N+N_{0}\right) M P a \sqrt{m} \\
K_{f C}(N)=98-1,3 \cdot 10^{-2}\left(N+N_{0}\right) M P a \sqrt{m} .(7)
\end{gathered}
$$

$N_{0}$ - initial operating time (loading cycles number) of pipe time before gas pipeline residual lifetime estimation. When known value $K_{\text {Imax }}$ put in equation (6) and correlation (7) with numerical values of pipe geometry parameters and loading, we will get a new differential equation with vary coefficients and its integration is only possible when the numerical method is applied. Based on numerical results of problem solution graphical dependency of pipe residual life time on initial defect size $\rho_{0}$ and initial operation time $N_{0}$ are built and shown on Fig. 4. It is established, that the increase

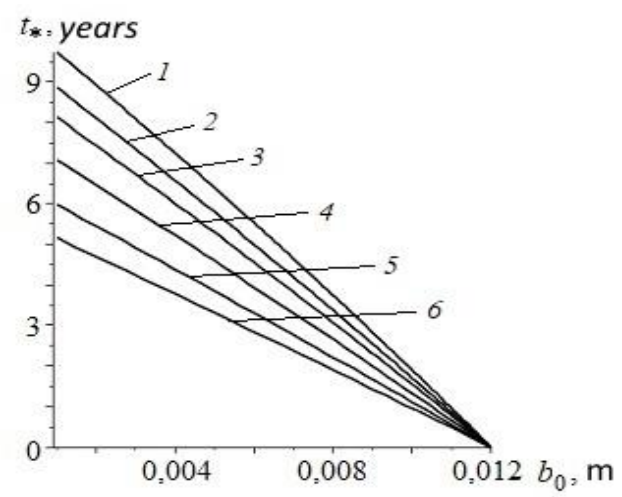

Fig.4. Dependence $t^{*} \sim b_{0}$ of pipe residual life time on initial crack depth $b_{0}$ and initial operation time $t_{0}: 1-\mathrm{t} 0=0 ; 2-4 ; 3-8 ; 4-15 ; 5-25 ; 6-35$ 
of the initial defect size and initial operation time essentially decreases oil pipeline residual lifetime.

\section{Evaluation of residual lifetime for X60 steel pipe of the oil pipeline at variable oil flow and metal hydrogenation.}

Consider oil pipeline pipe made of X60 steel with external surface semi-elliptical crack which is filled with soil corrosive environment and it is under hydrogenation. The geometry parameters of the pipe and its loading are the same as in the first case. The task consists in the determination of pipe residual life-time $N=N_{*}$ taking into account loading, corrosive media action, hydrogenation and steel operational. Fatigue crack growth diagrams for operated and reserved pipes which were obtained by Andeikiv and coauthors [9] are shown on a Fig 5. The plateau of constant crack growth rate $V$ with variable SIF range on this diagrams could be observed. This rate $V(N)$ for any operation time expressed in cycles $N$ could be approximately represented by linear dependency:

$$
V(N) \approx 10^{-7}\left[1,4+0,14\left(N_{0}+N\right)\right] \mathrm{m} / \text { cycle }(8)
$$

The task could be solved in the same way as in the. To determine pipe residual lifetime taking into account mentioned before parameters of its operation and material degradation following equations are obtained:

$$
\begin{gathered}
N_{*}=\sqrt{\left(10+N_{0}\right)^{2}+14,3 \cdot 10^{7}\left(0,0187-b_{0}\right)}-10-N_{0}, \text { cycle; } \\
t_{*}=0,015\left[\sqrt{\left(10+N_{0}\right)^{2}+14,3 \cdot 10^{7}\left(0,0187-b_{0}\right)}-10-N_{0}\right], \text { year. }(9)
\end{gathered}
$$

The graphical dependency of pipe residual lifetime $t_{*}$ on the initial crack depth $b_{0}$ and initial operation time $t_{0}$ are built by using Eq. (9) and shown on a Fig. 5 . It is established, that pipe residual life time essentially decrease with initial defect size and initial operation time increase.

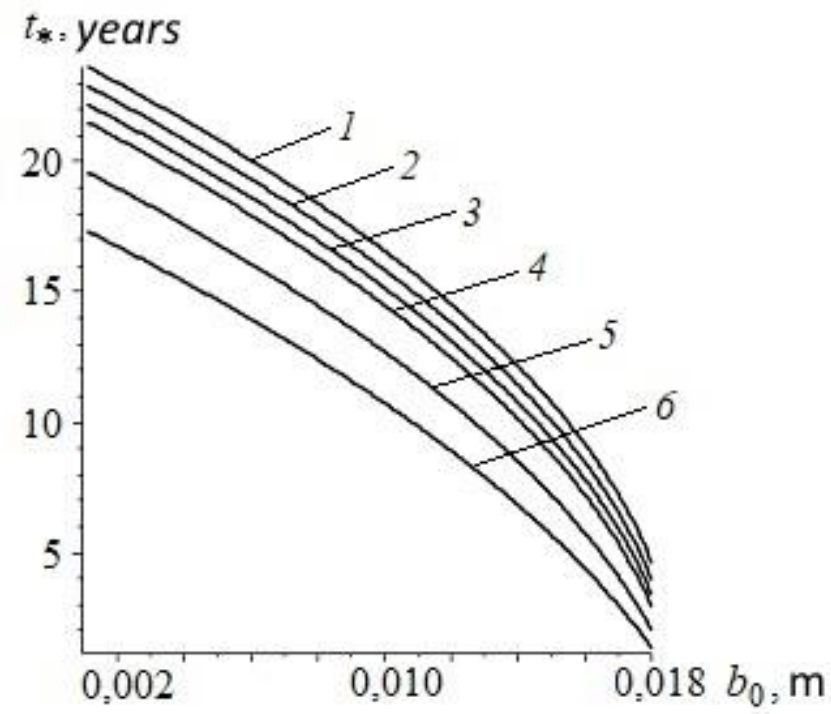

Fig.5. Dependency $t^{*} \sim b_{0}$ of pipe residual life time on initial crack depth $b_{0}$ and initial operation time $N_{0}: 1-N_{0}=0 ; 2-50 ; 3-100 ; 4-150 ; 5-300 ; 6-500$. 


\section{Conclusion}

The method of oil pipeline residual lifetime calculation is developed. It allows us to calculate the residual lifetime of pipe with surface cracks under the consistent action of long-term loading and corrosive-hydrogenating environment as well as taking into account operational material degradation, namely, characteristics of fatigue crack growth resistance changes during operation time as the most sensitive to metal state changes. It is also considering crack initiation after some period of pipe operation when an essential level of metal operational degradation is reached. It is shown that material degradation during pipeline operation essentially decreases pipe residual lifetime.

\section{References}

[1] Nykyforchyn H. M., Tsyrul'nyk O. T., Petryna D. Yu., Hredil' M. I. “Degradation of steels used in gas main pipelines during their 40-year operation," Strength of Materials, 2009, 41, № 5, pp. 501-505.

[2] Krasovskii A. Ya., Lokhman I. V., Orynyak I. V. „Stress-corrosion failures of main pipelines," Strength of Materials, 2012, 44, № 2, pp. 129-143.

[3] Kryzhanivs'kyi E. I., Hrabovs'kyi R. S., Mandryk O. M. „Estimation of the serviceability of oil and gas pipelines after long-term operation according to the parameters of their defectiveness," Materials Science, 2013, Vol. 49, № 1, pp. 117-123.

[4] Polishchuk L. K., Kharchenko H. V., and Zvirko O. I. "Corrosion-fatigue crack-growth resistance of steel of the boom of a clamp-forming machine," Materials Science, 2015, Vol. 51, № 2, pp. 229-234.

[3] Gabetta G., Nykyforchyn H. M., Lunarska E., Zonta P. P., Tsyrulnyk O. T., Nikiforov K., Hredil M. I., Petryna D. Yu., Vuherer T. "Effect of in-service degradation of trunk gas pipeline steel on its "in-bulk" properties," Materials Science, 2008, Vol. 44, № 1, - pp. 88-99.

[4] Andreikiv O. Ye., Skal's'kyi V. R., Opanasovych V. K., Dolins'ka I. Ya., Shtoiko I. P. "Determination of the period of subcritical growth of creep-fatigue cracks under block loading," Journal of Mathematical Sciences, 2017, Vol. 222, № 2, pp.103-113.

[5] Andreikiv O. E., Nykyforchyn H. M., Shtoiko I. P., Lysyk A. R. "Evaluation of the residual life of a pipe of oil pipeline with an external surface stress-corrosion crack for a laminar flow of oil with repeated hydraulic shocks," Materials Science, 2017, Vol. 53, № 2, pp. 216-226.

[6] Andreikiv O. E., Raiter O. K., Shtoiko I. P. "Determination of the period of subcritical growth of an internal surface stress-corrosion crack in a pipe of pipeline for the turbulent flow of oil with hydraulic shocks," Materials Science, 2018, Vol. 53, № 6, pp. 842-848

[7] Andreikiv O. E., Hembara O. V., Tsyrul'nyk O. T., Nyrkova L. I. "Evaluation of the residual lifetime of a section of a main gas pipeline after long-term operation," Materials Science, 2013, Vol. 48, N 2, pp. 231-238. 